Title:

QUARK AND PROTON SPIN STRUCTURE IN THE INSTANTON LIQUID MODEL OF QCD

$$
\text { CONF-970564- }
$$

Author(s):

Submitted to:

A. Blotz, Theoretical Division, Los Alamos National Laboratory, Los Alamos, NM 87545, USA

Edward Shuryak, State University of Stony Brook, Stony Brook, NY 11794, USA
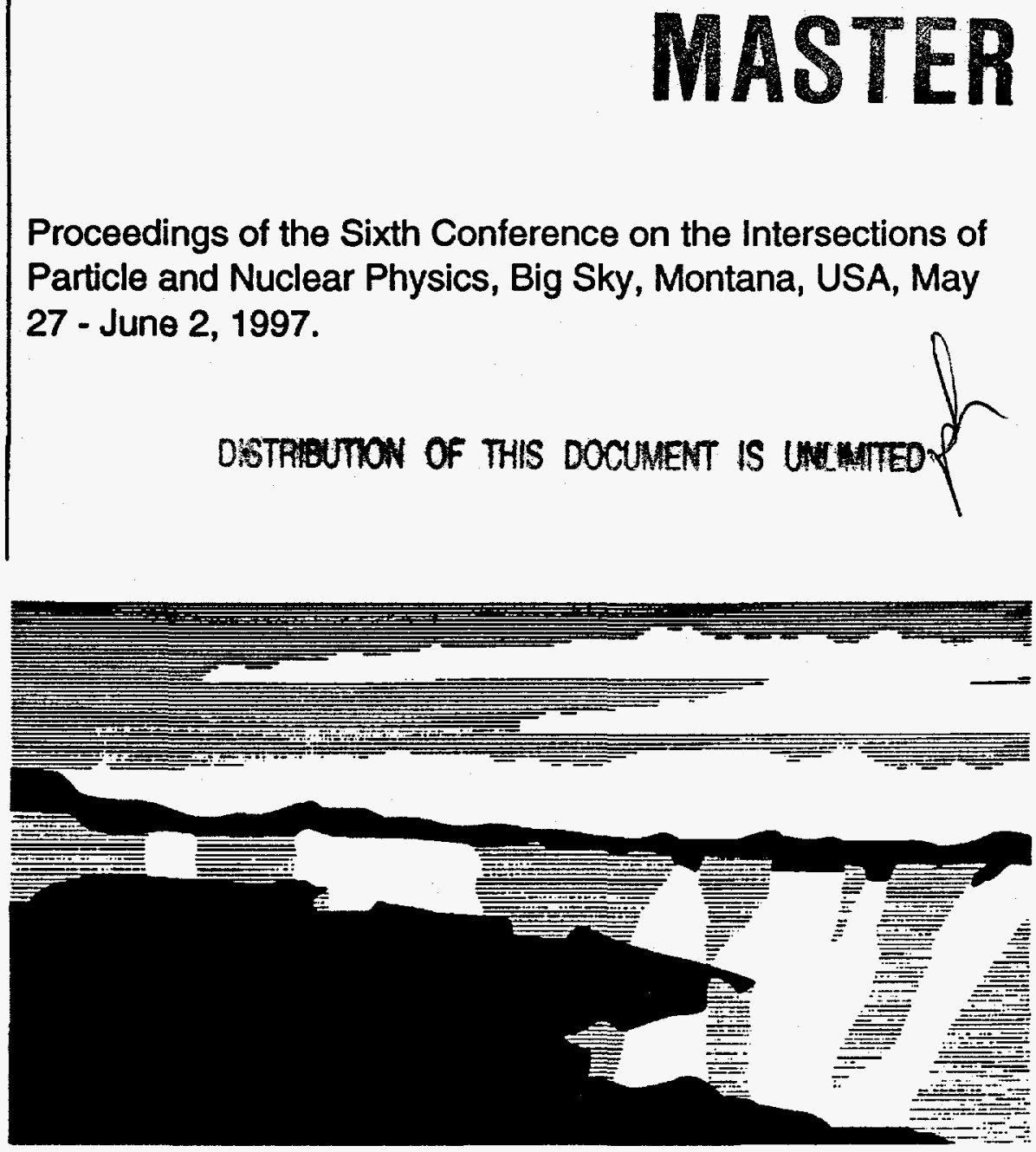

Los Alamos National Laboratory, en affirmative action/equal opportunity ermplóyer, is operated by the University of California for the U.S. Department of Energy under contract W-7405-ENG-36. By acceptance of this article. the publisher recognizes that the U.S. Government retains a nonexclusive, royalty-free license to publish or reproduce the pubished form of this contribution, or to allow others to do so, for U.S. Governmont purposes. The Los Alamos National Laboratory requests that the publisher identify this article as work pertormed under the auspices of the U.S. Department of Energy. 


\section{DISCLAIMER}

This report was prepared as an account of work sponsored by an agency of the United States Government. Neither the United States Government nor any agency thereof, nor any of their employees, makes any wartanty, express or implied, or assumes any legal liability or responsibility for the accuracy, completeness, or usefulness of any information, apparatus, product, or process disclosed, or represents that its use would not infringe privately owned rights. Reference herein to any specific commercial product, process, or service by trade name, trademark, manufacturer, or otherwise does not necessarily constitute or imply its endorsement, recommendation, or favoring by the United States Government or any agency thereof. The views and opinions of authors expressed herein do not necessarily state or reflect those of the United States Government or any agency thereof. 


\section{DESchamisR}

Portions of this docounent moy be illegible in electronic image produets. Images are produced from the best available origion docementert 


\title{
Quark and Proton Spin Structure in the Instanton Liquid Model of $\mathrm{QCD}^{1}$
}

\author{
Andree Blotz ${ }^{*}$ and Edward Shuryak ${ }^{\dagger}$ \\ ${ }^{*}$ Los Alamos National Laboratory, Los Alamos, NM 87545 \\ †State University of Stony Brook, Stony Brook, NY 11794
}

\begin{abstract}
Within an instanton based model of QCD we address the important question of how much of the proton spin is carried by the spins of the quarks and how much is due to orbital angular momentum and the spins of the gluons. Since this question arises already on the level of a single quark inside the proton, we study axial vector correlation functions for a quark in the so called Random Instanton Liquid Model (RILM) as well as for the Interacting Instanton Liquid Model (IILM).
\end{abstract}

\section{INTRODUCTION}

Recent lattice calculations support the decade old picture of a ground state of QCD which is filled with a liquid of instantons and anti-instantons. These classical solutions of Euclidean field equations do not only describe the qualitative features of low energy QCD but also quantitatively a large variety of hadronic and gluonic two-point correlation functions [?]. As a first step to investigate the structure of the hadrons we have calculated the three point correlation function of the pion with an external electro-magnetic field [?]. The result was not only the reproduction of the monopole shape formfactor but showed also very nicely that the formfactor of the pion is basically the formfactor of the instanton. Hereby the experimentally measured formfactor confirmed an average instanton size $\bar{\rho} \simeq 0.35 \mathrm{fm}$, which is the value used for more than a decade now [?].

Based on this successful model we are asking now where 'the proton really gets its spin' [?]. For this aim we calculate three point correlation function for the axial vector current in coordinate space and project onto the axial vector

1) Talk presented at Sixth Conference on the Intersections of Particle and Nuclear Physics, Big Sky, Montana, USA, May 27-June 2, 1997 
coupling as well as the induced pseudoscalar coupling constant [?]. To confirm the finding we also evaluate the divergence of the axial current, which in the chiral limit is given by the anomaly, within a quark state.

\section{THE QUARK PROPAGATOR}

The propagator of a single quark in the Instanton Liquid Model is shown in Fig. 1 in dependence of the quark separation $\mathrm{x}$ and for the spin flip and spin non-flip amplitude. Both curves are normalized to the free spin non-flip amplitude, so that the short distance behaviour of the spin non-flip amplitude reflects asymptotic freedom The figure shows a fit with a constant constituent quark mass (cqm), where the quark mass turns out to be $\simeq 300 \mathrm{MeV}$, but it neither describes the shorter distances $<1 \mathrm{fm}$ nor the larger ones. A reliable fit is obtained by assuming a momentum dependent quark mass. For small momenta then, the mass corresponds roughly to the constituent quark mass $\simeq 360-370 \mathrm{MeV}$, whereas for larger momenta it approaches zero.

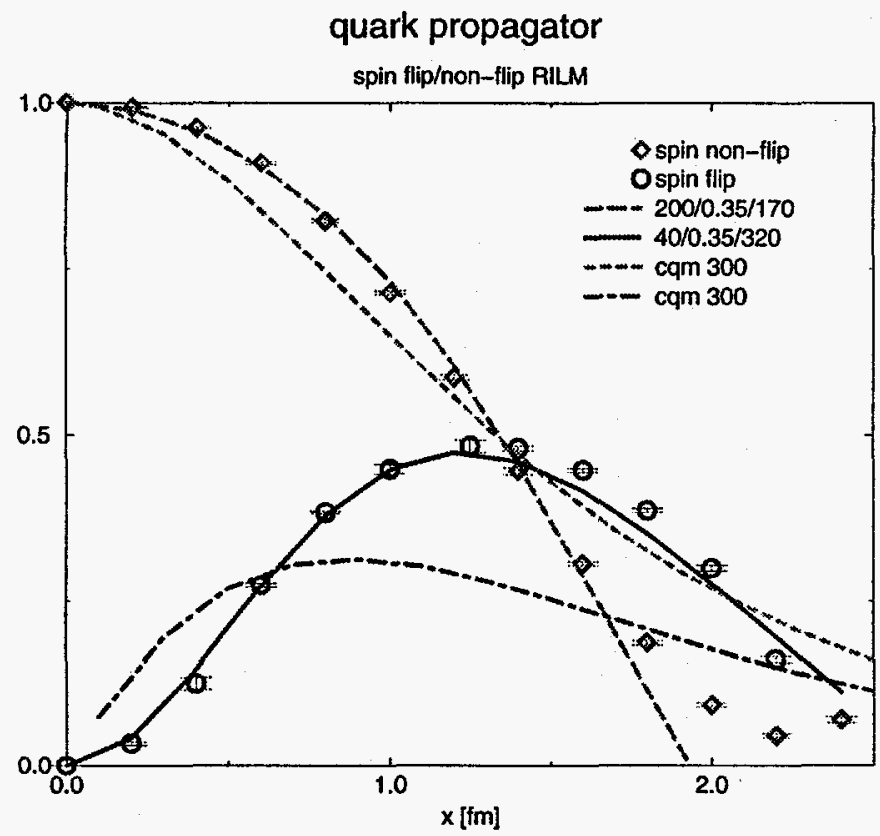

Fig. 1: The two-point correlation function of a quark, normalized to the free correlator and in the non interacting configuration (RILM). The instanton size is $\rho=0.35 \mathrm{fm}$ and the instanton density is $n=1 \mathrm{fm}^{-4}$ [5]. 


\section{THE DIVERGENCE OF THE AXIAL CORRELATOR}

Using this momentum dependent quark propagator one can determine the axial vector coupling $g_{A}$ either from axial vector current correlator [5] or from the anomaly. This is because the divergence of the flavor singlet axial current in QCD is non-vanishing in the chiral limit due to

$$
\partial_{\mu} j_{\mu, 5}^{G I}(x)=2 m \bar{q} i \gamma_{5} q+\frac{N_{f}}{16 \pi^{2}} G_{\mu \nu} \tilde{G}_{\mu \nu}
$$

In Fig. 2 we have evaluated the anomaly term $G_{\mu \nu} \tilde{G}_{\mu \nu}$ as well as the connected and disconnected contribution to $\bar{q} i \gamma_{5} q$ for a constituent quark. As can be seen the anomaly term (GG disc) almost cancels with the disconnected quark term ( $\mathrm{mP}$ disc) and moreover the total contribution of all 3 terms has a different sign than the anomaly term.

divergence of axial current

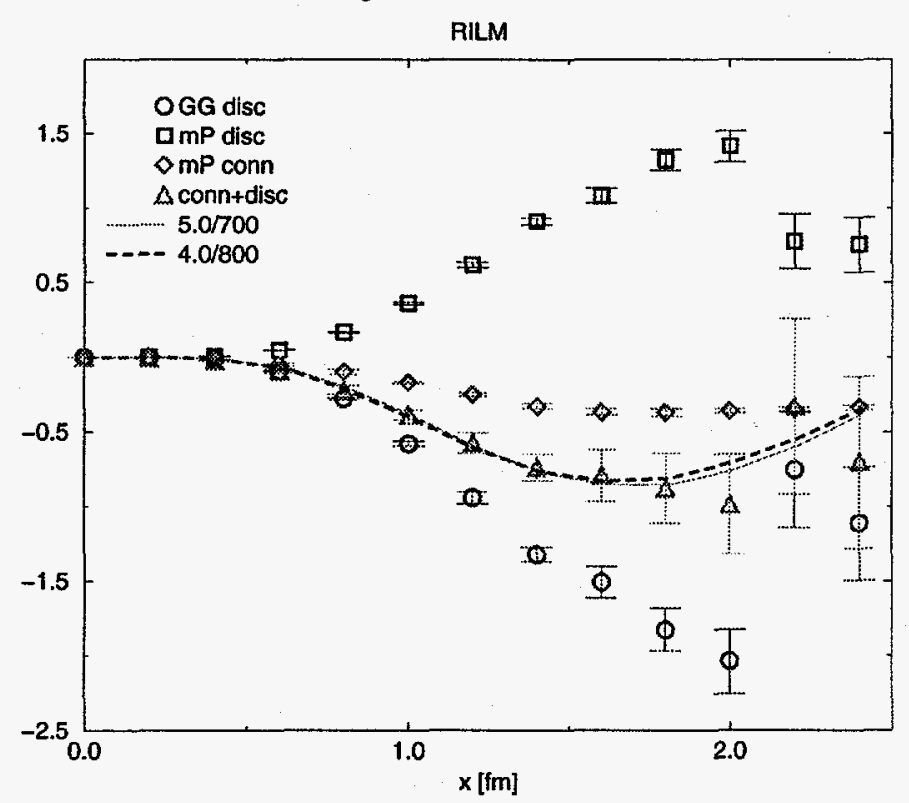

Fig. 2: The three-point correlation function $\Pi_{3}^{-}(x, y)$ of a quark, normalized to the free correlator and for the random configuration (RILM).

The final value of the quark spin expectation value from this calculation depends on the interactions of the instantons. Whereas random configurations of instantons and anti-instantons suggest that $g_{A}$ is indeed close to one, the interacting ensemble $I I L M$ shows a reduction of almost $50 \%$. This is an clear indication that instanton effects might explain the proton spin puzzle. 


\section{REFERENCES}

1. T. Schaefer and E. Shuryak, Instantons in QCD, HEP-PH 9610451, 1996.

2. A. Blotz and E. Shuryak, Phys. Rev. D55, 4055 (1997).

3. E. V. Shuryak, the QCD Vacuum, Hadrons and the Superdense Matter, World Scientific, Singapore, 1988.

4. R. L. Jaffe, Phys. Today 48, NO.924 (1996).

5. A. Blotz and E. Shuryak, Structure of a constituent quark in the instanton liquid, 1997. 